\title{
Study of D-band LTCC Leaky Wave Antenna Optimized for Broadside Radiation
}

\author{
Przemyslaw PIASECKI, Yevhen YASHCHYSHYN \\ Inst. of Radio Electronics and Multimedia Technology, Warsaw University of Technology, \\ Nowowiejska Street 15/19, 00-665 Warsaw, Poland \\ \{p.piasecki, y.yashchyshyn\}@ire.pw.edu.pl
}

Submitted November 1, 2017 / Accepted January 8, 2018

\begin{abstract}
This paper presents the design, fabrication and measurements of a Low Temperature Co-Fired Ceramic leaky wave antenna optimized for broadside radiation. The antenna consists of two DuPont $9 k 7$ material layers. The thickness of each layer is approximately $115 \mu \mathrm{m}$. The bottom layer plays the role of the main substrate and a top layer is used for creating a corrugated structure. The developed antenna operates in the frequency range of $125 \mathrm{GHz}-135 \mathrm{GHz}$ with a minimized radiation pattern squint below 8 degrees in the whole operating bandwidth. The antenna reflection coefficient $S_{11}$ is less than $-10 \mathrm{~dB}$ in the operating frequency bandwidth and the maximum measured antenna gain is $10 \mathrm{dBi}$. Moreover a study of different antenna radiating structures and their capability of broadside radiation and reflection coefficient properties are discussed.
\end{abstract}

\section{Keywords}

Antenna, leaky wave, LTCC, mm-waves

\section{Introduction}

Recently, there has been substantial effort devoted to the development of antennas that would cover the millimeter wave range, leading to a sharp increase of many technologies in this frequency range. It is a big challenge to implement antenna design beyond $100 \mathrm{GHz}$ because the uncertainties and tolerances of the fabrication process as well as material characteristics both affect antenna performance more significantly. Moreover, a very important issue for system cost reduction is the integration of the antenna with other components in a wireless millimeter waves system.

Taking into account the aforementioned requirements, Low Temperature Co-fired Ceramic Technology (LTCC) can be considered a promising technology for manufacturing mm-wave antennas. This technology has been developed over many years and is usually used in devices operating at frequencies below $100 \mathrm{GHz}$. The main advantages of LTCC technology are: a 3D multilayer structure, very good control of technological aspects and obtaining precise repeatability, flexibility in the number of layers and kinds of materials used, the possibility of integrating lumped and distributed elements, and also the possibility of on-chip mounting by using flip-chip or wire-bonding techniques. Moreover the thermal coefficient of LTCC materials is comparable to semiconductor structures, which implies a high density of mounting integrated circuits in a small area.

In recent years, a few papers have described LTCC antennas and array antennas operating in the frequency range above $100 \mathrm{GHz}$ [1-5]. However, most of the presented approaches are complicated, especially through the use of many vias, which greatly increase the manufacturing cost. Moreover, there is a problem with antenna efficiency due to antenna design and material losses. Considering the problems mentioned relating to the frequency range above $100 \mathrm{GHz}$, we propose the LTCC leaky wave antenna as a good candidate for this range.

Generally, leaky wave antennas are used for many applications in which the functionality of frequency scanning can be useful [9], [10]. The antenna radiation pattern is generated by a current distribution, which is obtained by setting the proper distance between metallic plates on the top of the LTCC material layer. However, sometimes the option of obtaining broadside radiation, especially in a broadband frequency range, is demanded. The main problem of obtaining the broadside radiation in leaky wave antennas is the occurrence of increased Voltage Standing Wave Ratio (VSWR) [7]. In order to decrease the effect of higher VSWR level, an irregular distance between neighboring radiators has been proposed [7], [8]. Also, this antenna consists of only two LTCC layers which is a very low cost solution in comparison to antennas documented in the literature [1-5]. Moreover, in this type of antenna radiating structure, special vias are not required to ensure proper antenna signal excitation.

In this paper, the results of the study of a low cost D-band LTCC leaky wave antenna optimized for broadside radiation are presented and discussed. To the authors' best knowledge, such type of manufactured and measured LTCC leaky wave antenna operating in the frequency 
range above $100 \mathrm{GHz}$ has not been extensively investigated so far. The antenna can be integrated in a simple way with other passive and active components of a wireless system.

\section{Design and Manufacture of LTCC Antenna}

The principle of operation a leaky wave antenna can be considered as a linear array of periodic radiating elements [8] where the main beam direction is described as:

$$
\theta=\arcsin \left(\frac{\beta_{\mathrm{d}}}{k_{0}} \cdot\left(1-\frac{\lambda_{\mathrm{d}} \cdot n}{d}\right)\right)
$$

where $k_{0}$ is the free space wavenumber, $\beta_{\mathrm{d}}$ is the phase constant in a dielectric substrate, $\lambda_{\mathrm{d}}$ is the wavelength in a dielectric substrate, $d$ is the distance between radiators, $n$ $=0, \pm 1, \pm 2, \pm 3, \ldots$

Taking into consideration the possibility of using LTCC technology in the frequency range above $100 \mathrm{GHz}$, certain assumptions have been made: the utilization of commonly available LTCC materials, the simplicity of structure design and the possibility of integration of manufactured antennas with a proper transition in order to measure antenna characteristics. Based on these requirements, the antenna has been designed using the LTCC DuPont $9 \mathrm{k} 7$ material. After a firing process the single layer thickness is approximately $115 \mu \mathrm{m}$. The properties of this ceramic material have been measured in the $90 \mathrm{GHz}$ to $140 \mathrm{GHz}$ frequency band with a special measurement setup [11]. The measured relative electrical permittivity of the material is $\varepsilon_{\mathrm{r}} \approx 7.35$ and the loss tangent is $\tan \delta \approx 0.01$.

The appropriate choice of radiating structure depends on several factors, e.g. LTCC material properties, thickness of a single material layer, capabilities of manufacture technology and so on [12], [13]. In Fig. 1, five structures are shown which have been taken into consideration during the design process of the antenna. One of the structures (Fig. 1.a) consists of one material layer only and the others (Fig. 1.b-e) are designed with two layers of LTCC. All structures are fed by waveguide port at the beginning of the structure to provide a surface wave source in order to feed the antenna aperture. The effects of distortion on the radiation pattern produced by the waveguide transition have not been considered in this part of the simulation. The results of this experiment have shown the influence of the number of LTCC layers and radiating structure pattern on the radiation pattern and the possibility of broadside radiation.

Each of the structures has been optimized to achieve the minimal requirements: broadside radiation and $S_{11}$ below $-10 \mathrm{~dB}$. The first structure (Fig. 1.a) consists of only one layer of LTCC material of thickness $h_{1 \text { layer }}=115 \mu \mathrm{m}$. On top of this layer regular metalized strips have been placed. This kind of structure was the simplest antenna to have been considered, but unfortunately such a thin LTCC a)

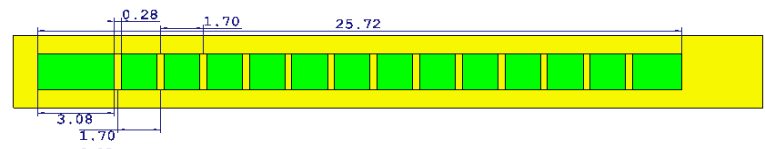

b)

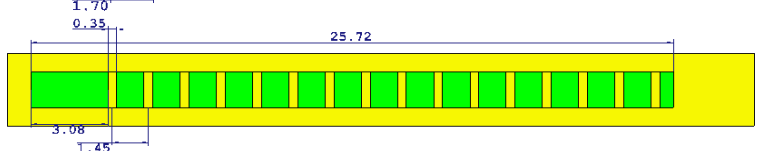

c)

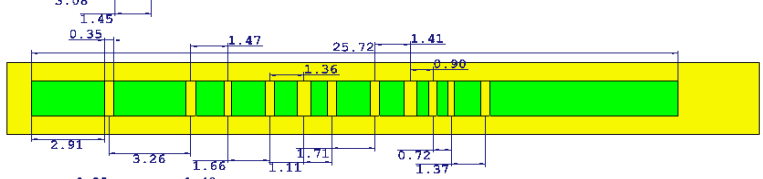

d)

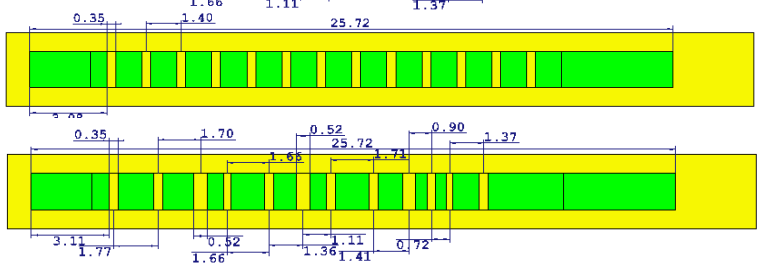

Fig. 1. Different radiating structures of the designed antenna: a) 1 layer LTCC plane, regular radiating structure, b) 2 layers LTCC plane, regular radiating structure, c) 2 layers LTCC plane, irregular radiating structure, d) 2 layers LTCC corrugated, regular radiating structure, e) 2 layers LTCC corrugated, irregular radiating structure.

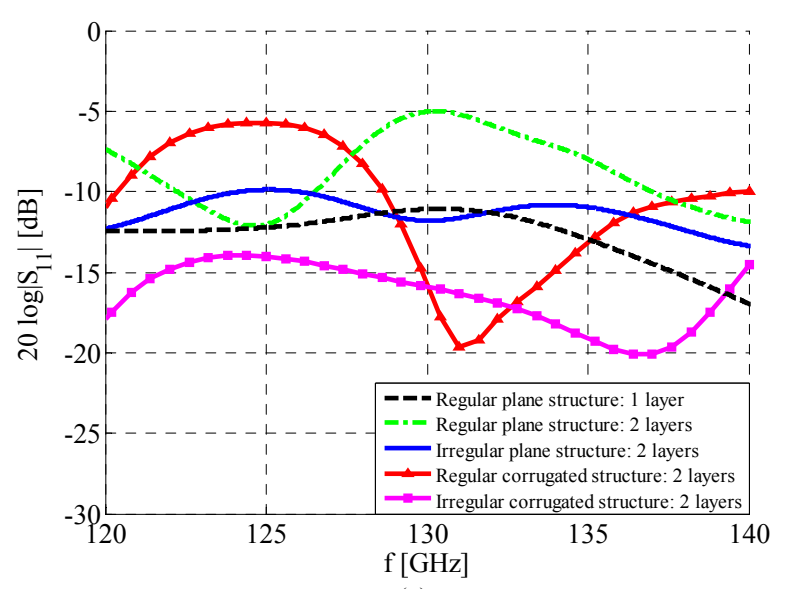

(a)

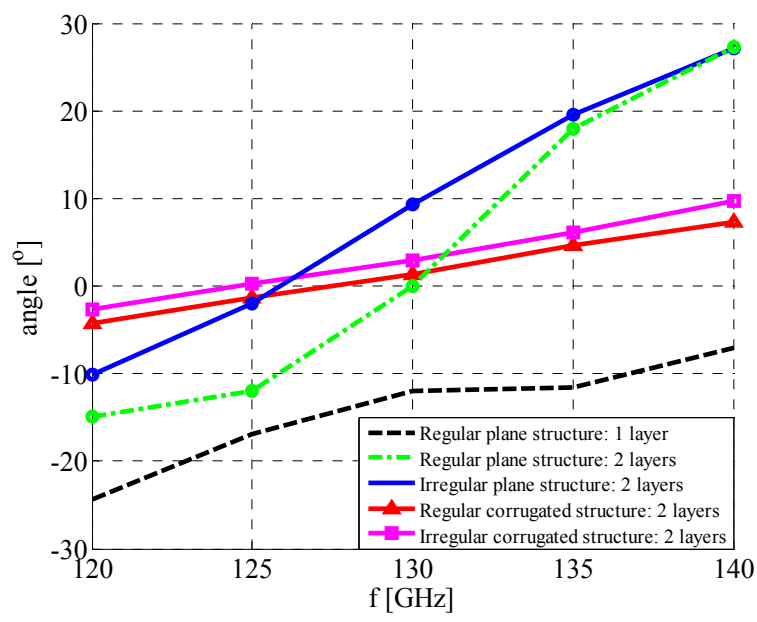

(b)

Fig. 2. A comparison between: a) regular and irregular structure dependencies of $\mathrm{S}_{11}, \mathrm{~b}$ ) the main beam direction in the E plane. 


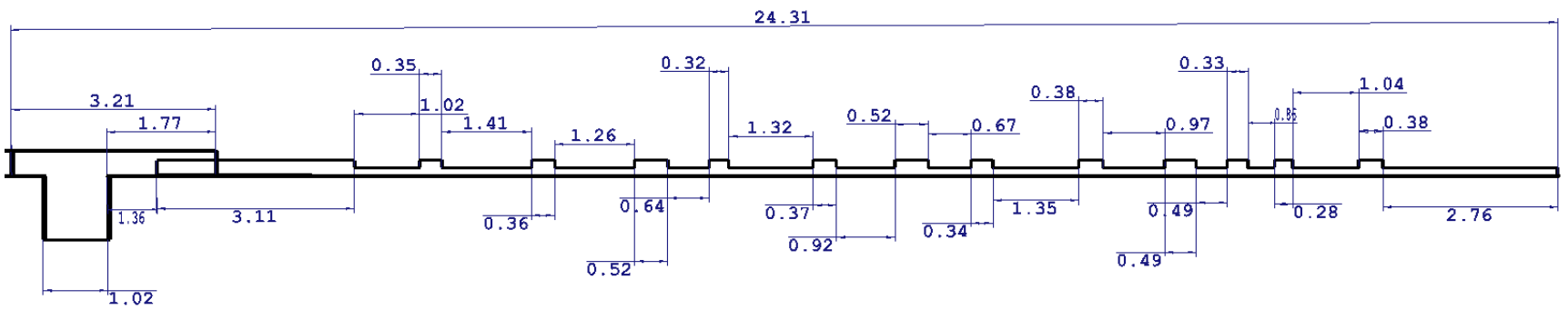

Fig. 3. Side view of the antenna radiated irregular structure and waveguide transit.

layer was not able to meet the requirement of broadside radiation. The simulated results of $\mathrm{S}_{11}$ and the main beam direction for each radiating structure are presented in Fig. 2.a) and Fig. 2.b), respectively.

Due to the failure of sufficient broadside radiation, the single LTCC layer antenna was rejected for further consideration. The second (Fig. 1.b) and the third (Fig. 1.c) structures consist of two plane LTCC layers and metalized regular and irregular deployed strips. As can be seen in Fig. 2(b), the direction of the main beam of each structure is similar. However, the levels of the reflection coefficients are not satisfactory, especially in a case of broadside radiation. The effect of higher VSWR is caused by the constructive interference of in-phase signals reflected from the regular structure of metalized strips. The irregular placement of metalized strips allows for a lower $\mathrm{S}_{11}$ in a wider frequency range and also minimization of the beam scanning effect in the antenna operating frequency. However, the main beam direction in the cases shown in Fig. 1.b) and Fig. 1.c) varies approximately from $20^{\circ}$ to $30^{\circ}$, respectively, which is not a satisfactory result. Moving forward, the fourth (Fig. 1.d) and fifth (Fig. 1.e) structures have been additionally corrugated which further improved $S_{11}$ and also minimized the main beam scanning in a broadband frequency. The best results were obtained for the irregular corrugated structure with metalized corrugated layer, which is depicted in Fig. 1.e). The final simulation of the antenna with a proper radiating structure and the waveguide transition was carried out and the dimensions of the optimized antenna are shown in Fig. 3.

In Fig. 4 the simulated radiation pattern in both $\mathrm{E}$ and $\mathrm{H}$ planes are depicted. The main beam of the radiation pattern is near the broadside radiation of the antenna. In the $10 \mathrm{GHz}$ bandwidth range from $125 \mathrm{GHz}-135 \mathrm{GHz}$, the main antenna beam scanning is less than $8^{\circ}$ in the $\mathrm{E}$ plane. The average $3 \mathrm{~dB}$ beam width in the operating bandwidth is approximately $9^{\circ}$ which means that even though the antenna beam squint is $8^{\circ}$, the scanned area is always in the $3 \mathrm{~dB}$ beamwidth. The gain computed in the simulated antenna for frequencies $125 \mathrm{GHz}, 130 \mathrm{GHz}$ and $135 \mathrm{GHz}$ has been obtained as follows: $\mathrm{G}_{125}=12.3 \mathrm{dBi}$, $\mathrm{G}_{130}=12.0 \mathrm{dBi}, \mathrm{G}_{135}=11.5 \mathrm{dBi}$, respectively. Depending on the frequency, the side lobe level (SLL) is below $6 \mathrm{~dB}$ in the worst case and for the middle frequency the SLL is better than $10 \mathrm{~dB}$. The radiation pattern in the $\mathrm{H}$ plane is wider than in the E plane which is caused by the narrow antenna radiating structure in the $\mathrm{H}$ plane.

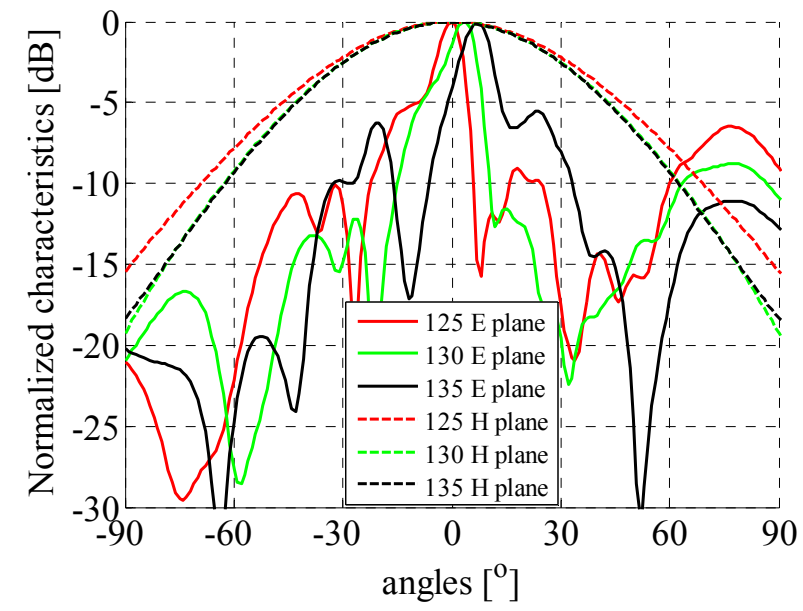

Fig. 4. Simulated radiation pattern of the investigated leaky wave antenna.

a)

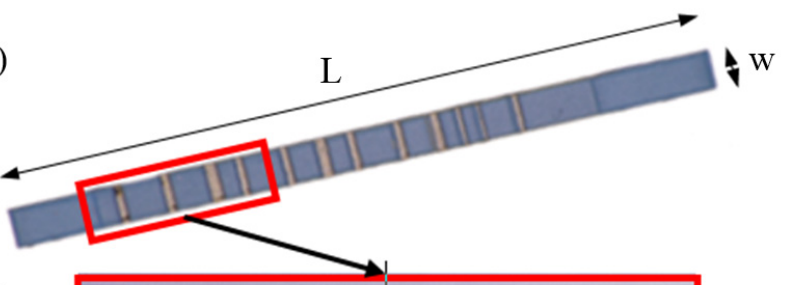

b)

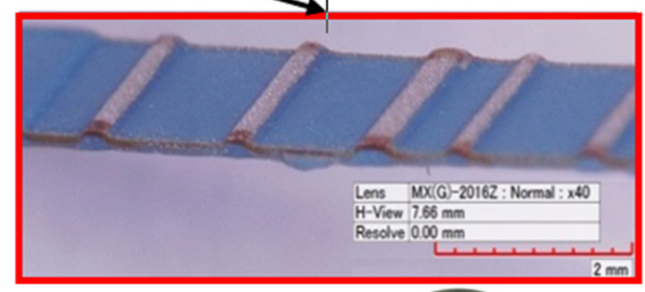

c)

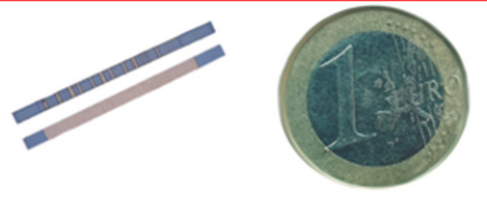

Fig. 5. Manufactured LTCC leaky wave antenna.

The designed structure was manufactured by laser embossing green ceramic sheets, laminating and co-firing the two prepared layers. In the last step, printing of conductive paths on top of the corrugated layer using ESL 9916 paste was performed [14]. The thickness of the top metal layer obtained by screen-printing technique is approximately $10 \mu \mathrm{m}$. The manufactured antenna dimensions ( $L=26.40 \mathrm{~mm}, w=1.47 \mathrm{~mm}$ ), a zoomed-in part of the structure, and size comparison with $1 €$ coin, are depicted in Fig. 5.a), b) and c), respectively. 
In Fig. 5.b) some bleeding on the edge of bar structures can be seen. This is the effect of melting the LTCC material during the firing process. Also, shrinkage of the DuPont material [13] may affect the dimensions of the radiating structure. In order to investigate the manufactured physical leaky wave antenna, a suitable measurement setup was used for obtaining the antenna radiation pattern measurement results. These results are presented in the next section.

\section{Measurement Results}

In this section, the measurement results of the fabricated antenna are presented. The measurement setup includes: vector network analyzer, two frequency extenders, which operate in the $90 \mathrm{GHz}-140 \mathrm{GHz}$ frequency band, a rotating table, positioners for manually moving the extenders in five axes. By using twisted waveguides it is possible to measure an antenna radiation pattern in two orthogonal polarizations, the $\mathrm{E}$ and $\mathrm{H}$ planes. The rotary table position is in the range of $-90^{\circ}$ to $+90^{\circ}$. In the current configuration, the rotational accuracy of the positioner is less than $0.1^{\circ}$. The positioning accuracy of the frequency extender is one micrometer in all possible directions.

The LTCC leaky wave antenna is fed using a special transition, which was designed and manufactured for this study (Fig. 6). The transition consists of two parts: a main plate (Fig. 6.a), which is the base for the antenna radiating structure and the second part, a cover with holes (Fig. 6.b) to screw the whole transition to the end of the frequency extender waveguide.

Figure 7 shows three measurement scenarios of the leaky wave antenna mounted to the transition waveguide output: Fig. 7.a) - the antenna is placed on an uncovered metal plate, Fig. 7.b) - the metal plate with stuck absorber and Fig. 7.c) - the antenna with the narrow metal plate.

In Fig. 8 the simulated and measured reflection coefficients of the designed antenna are illustrated, which agree well. The radiation pattern in the $\mathrm{H}$ plane is in accordance with the simulations which is shown in Fig. 9. Figures 10 to 12 present the radiation patterns in the $\mathrm{E}$ plane, where the $\mathrm{x}$ axis shows the operating antenna frequency and, in the $y$ axis, the direction of the main antenna beam is presented. The comparison with Fig. 2 shows similarity of the main beam squint in the frequency range. The dependency of antenna radiation squint close to broadside direction can be observed.

The presented radiation patterns for three antenna scenarios (Fig. 7) show, that changing the closest radiating structure environment implies differences in measured characteristics. The main beam direction is very close in all cases, and a little shifted according to broadside radiation. This is caused by different effective material permittivity due to sticking the antenna radiating structure to the metal plate. The radiation pattern beyond the main beam is slightly changed. These changes are affected by different a)

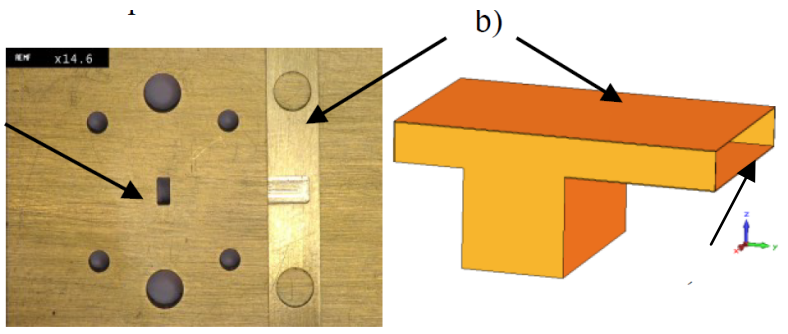

Fig. 6. Manufactured WR-8 waveguide transition to the radiating antenna structure.

a)

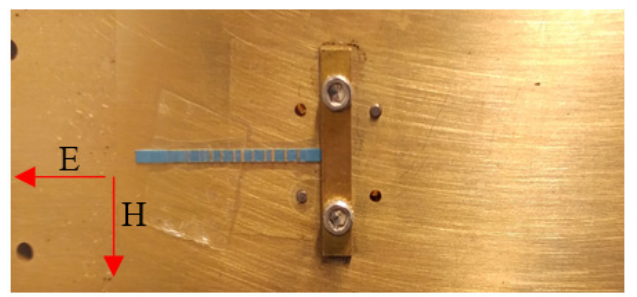

b)

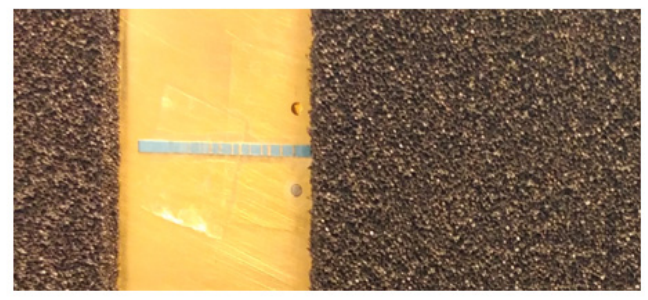

c)

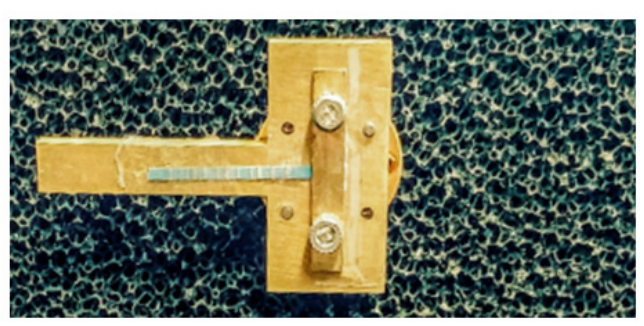

Fig. 7. The leaky wave antenna mounted in three scenarios: a) to an uncovered metal plate, b) to the metal plate with stuck absorber, c) to the cut off part of the metal plane.

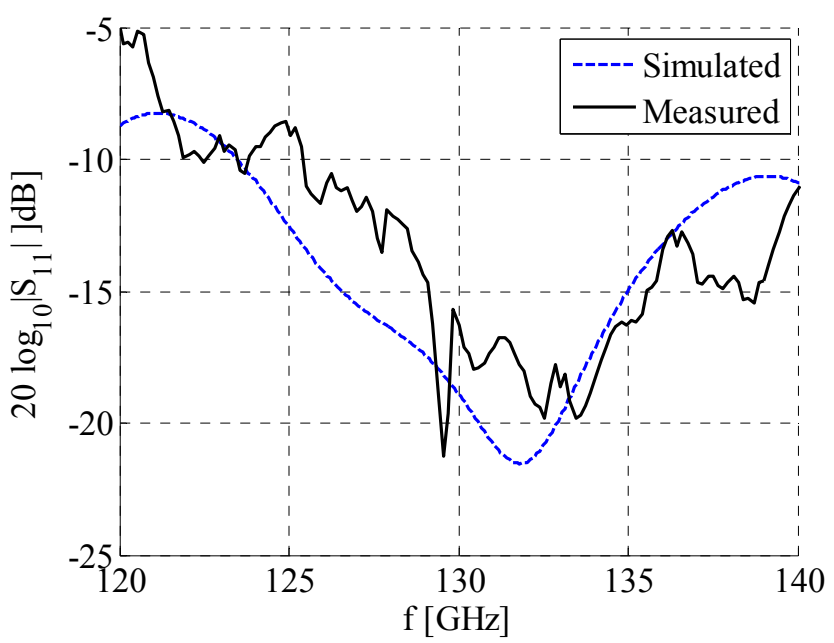

Fig. 8. The antenna reflection coefficient $S_{11}$ for the scenarios shown in Fig. 7.a). 


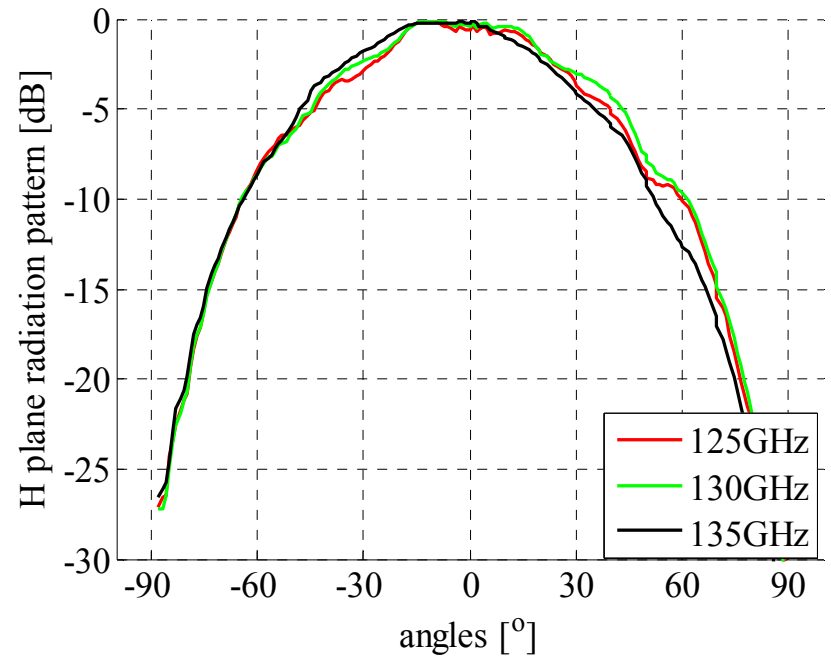

Fig. 9. The measurement results of the $H$ plane radiation pattern.

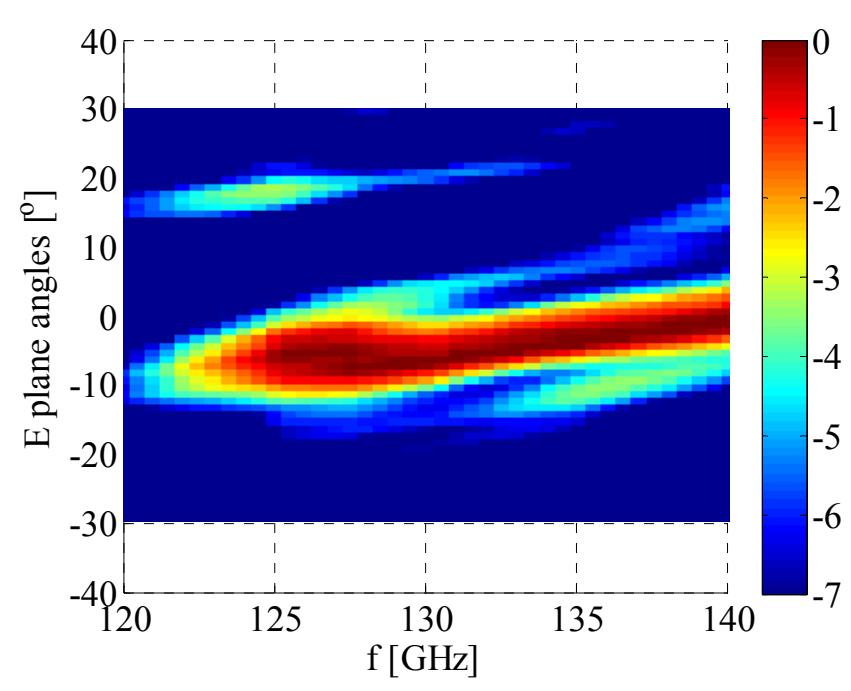

Fig. 10. The E plane antenna pattern in dependency of frequency range shown for the scenario in Fig. 7.a).

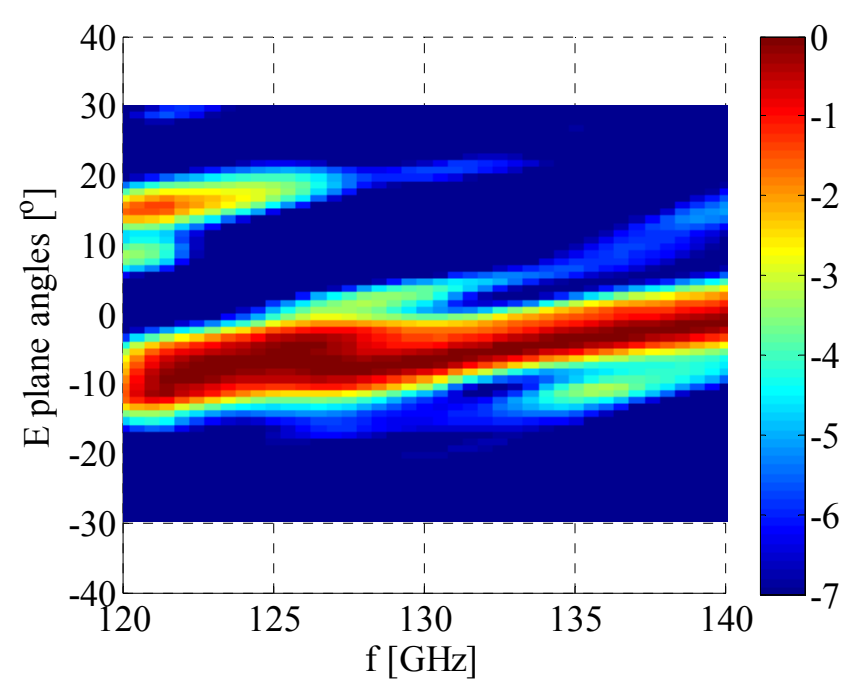

Fig. 11. The E plane antenna pattern in dependency of frequency range shown for the scenario in Fig. 7.b).

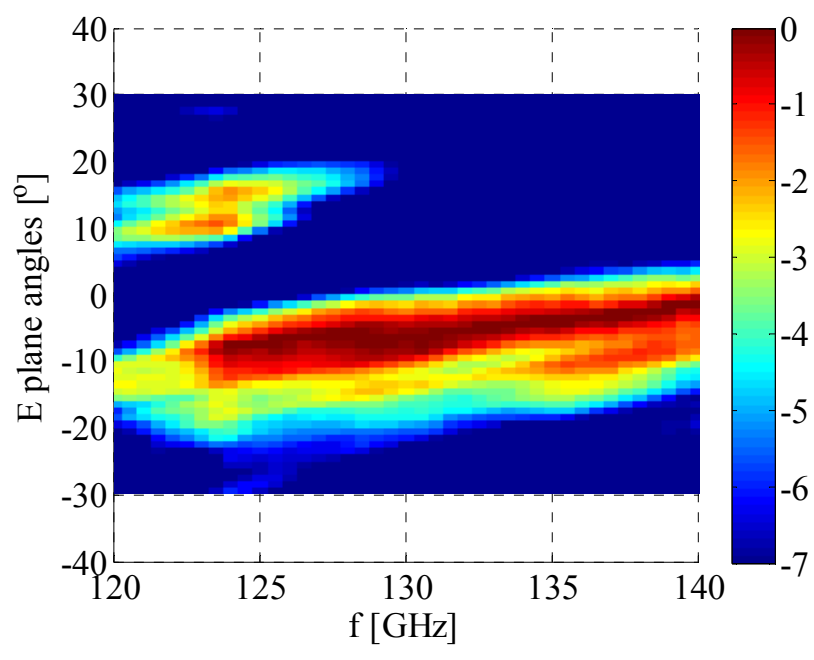

Fig. 12. The E plane antenna pattern in dependency of frequency range shown for the scenario in Fig. 7.c).

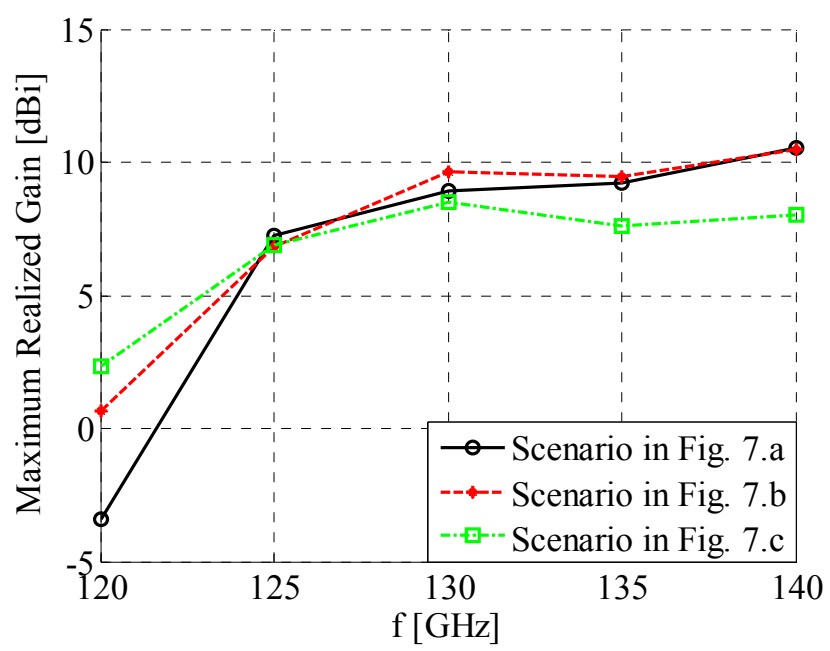

Fig. 13. Measured antenna gain for the scenarios shown in Fig. 7.

antenna mountings on metal or with absorber covered (Fig. 7). These facts should be taken into account during integration of the antenna with other millimeter wave components and packaging processes.

Figure 13 shows the maximum realized antenna gains for the operating bandwidth in the range of $\mathrm{E}$ plane angles between $-30^{\circ}$ and $+30^{\circ}$. The minimum measured gain is greater than $7 \mathrm{dBi}$.

\section{Conclusion}

In this paper, the investigation of an LTCC broadside antenna operating in the $125 \mathrm{GHz}$ to $135 \mathrm{GHz}$ range has been presented. The obtained measurement results show a good agreement with simulated results. The reflection coefficient $\mathrm{S}_{11}$ is less than $-10 \mathrm{~dB}$ in the operating bandwidth, the main beam squint is below 8 degrees and the minimum measured gain is greater than $7 \mathrm{dBi}$. 
Also, the paper shows the differences in radiation patterns, when different structures are present in the antenna's environment (Fig. 7). Moreover, the design of the antenna under study has allowed for testing of LTCC technology implemented in an antenna operating in the range of over $100 \mathrm{GHz}$, which has not been thoroughly investigated in leaky wave antennas so far. The possibility of manufacturing the designed antenna in this frequency range is very optimistic for future communication systems operating beyond $100 \mathrm{GHz}$, where LTCC technology would be used.

\section{Acknowledgments}

This work was partly supported by The National Center for Research and Development (NCBiR) in Poland, contract no. PBS3/A3/18/2015.

\section{References}

[1] XU, J., CHEN, Z. N., QING, X., et al. 140-GHz TE20-mode dielectric-loaded SIW slot antenna array in LTCC. IEEE Transactions on Antennas and Propagation, 2013, vol. 61, no. 4, p. 1784-1793. DOI: 10.1109/TAP.2012.2220317

[2] XU, J., CHEN, Z. N., QING, X. 270-GHz LTCC-integrated high gain cavity-backed Fresnel zone plate lens antenna. IEEE Transactions on Antennas and Propagation, 2013, vol. 61, no. 4, p. 1679-1687. DOI: 10.1109/TAP.2012.2232261

[3] ZHANG, B., GULAN, H., ZWICK, T., et al. Integration of a $140 \mathrm{GHz}$ packaged LTCC grid array antenna with an InP detector. IEEE Transactions on Components, Packaging and Manufacturing Technology, 2015, vol. 5, no. 8, p. 1060-1068. DOI: 10.1109/TCPMT.2015.2453407

[4] Bhutani, A., Gulan, H., GOETtel, B., et al. $122 \mathrm{GHz}$ aperture-coupled stacked patch microstrip antenna in LTCC technology. In Proceedings of the 10th European Conference on Antennas and Propagation EuCAP. Davos (Switzerland), 2016, 5 p. DOI: $10.1109 /$ EuCAP.2016.7481147

[5] TAJIMA, T., SONG, H., AJITO, K., et al. 300-GHz step profiled corrugated horn antennas integrated in LTCC. IEEE Transactions on Antennas and Propagation, 2014, vol. 62, no. 11, p. 5437 to 5444. DOI: 10.1109/TAP.2014.2350520

[6] MONTICONE, F., ALÙ, A. Leaky-wave theory, techniques, and applications: From microwaves to visible frequencies. IEEE Proceedings, 2015, vol. 103, no. 5, p. 793-821. DOI: 10.1109/JPROC.2015.2399419

[7] YASHCHYSHYN, Y., MODELSKI, J. W. Rigorous analysis and investigations of the scan antennas on a ferroelectric substrate. IEEE Transactions on Microwave Theory and Techniques, 2005, vol. 53, no. 2, p. 427-438. DOI: 10.1109/TMTT.2004.840779

[8] SOLBACH, K. E-band leaky wave antenna using dielectric image line with etched radiating elements. In IEEE MTT-S International Microwave Symposium Digest. Orlando (FL, USA), 1979, p. 214 to 217. DOI: 10.1109/MWSYM.1979.1124023

[9] YANG, S., LING, H. Application of a microstrip leaky wave antenna for range-azimuth tracking of humans. IEEE Geoscience and Remote Sensing Letters, 2013, vol. 19, no. 6, p. 1384-1388. DOI: 10.1109/LGRS.2013.2243401

[10] YASHCHYSHYN, Y., MODELSKI, J. Reconfigurable semiconductor antenna. In Proceedings of the $9^{\text {th }}$ International Conference The Experience of Designing and Application of CAD Systems in Microelectronics CADSM. Lviv-Polyana (Ukraine), 2007, p. 146-150. DOI: 10.1109/CADSM.2007.4297507

[11] ANDRUSHCHAK, N. A., KARBOVNYK, I. D. GODZISZEWSKI, K., et al. New interference technique for determination of low loss material permittivity in the extremely high frequency range. IEEE Transactions of Instrumentation and Measurement, 2015, vol. 64, no. 11, p. 3005-3012. DOI: 10.1109/TIM.2015.2437631

[12] PIASECKI, P. YASHCHYSHYN, Y., DENISOV, A. Investigation of LTCC leaky wave antenna operated in mm-wave band. In Proceedings of the 21th International Conference on Microwave, Radar and Wireless Communications MIKON. Krakow (Poland), 2016, 4 p. DOI: 10.1109/MIKON.2016.7492006

[13] YASHCHYSHYN, Y., BAJURKO, P. R., PIASECKI, P., et al. Experience in developing LTCC technologies for mm-wave antennas. In Proceedings of the $11^{\text {th }}$ European Conference on Antennas and Propagation EUCAP. Paris (France), 2017, 5 p. DOI: 10.23919/EuCAP.2017.7928083

[14] SYNKIEWICZ, B., KULAWIK, J., SKWAREK, A., et al. High resolution patterns on LTCC substrates for microwave applications obtained by screen printing and laser ablation. In Proceedings of the $39^{\text {th }}$ International Spring Seminar of Electronics Technology ISSE. Pilsen (Czech Republic), May 2016, p. 17-21. DOI: 10.1109/ISSE.2016.7563153

\section{About the Authors ...}

Przemyslaw PIASECKI was born in Grójec, Poland, in 1989. He received the M.Sc. degree in Telecommunication from the Warsaw University of Technology (WUT), Warsaw, Poland in 2013. He is currently working towards his Ph.D. degree in the Institute of Radioelectronics, Warsaw University of Technology and also working in PIT-Radwar company in the Department of Microwave Passive Components and Antenna Systems. His research interests include millimeter-wave antennas, LTCC technology, antenna measurements, beamforming technique and antennas for radar systems.

Yevhen YASHCHYSHYN was born in Lviv, Ukraine, in 1957. He received the M.Sc. degree in Radioelectronics Engineering from the Lviv University of Technology (Ukraine) in 1979, the Ph.D. degree in Microwave Devices and Antennas from the Moscow Institute of the Electronic and Mathematics in 1986, the D.Sc. degree (habilitation) degrees in Telecommunication from the Warsaw University of Technology (WUT) in 2006. In 2017 he obtained the state title of Professor. Since 1999, he has been with the Institute of Radioelectronics and Multimedia Technology of WUT. His research interests are: antenna theory and techniques, smart beamforming, reconfigurable antennas for communication and radar systems, and microwave photonics. 